\title{
Explosion Characteristics of Syngas/air Premixed Flames
}

\author{
Manh-Vu Tran', Gianfranco Scribano ${ }^{2}$ \\ ${ }^{1}$ School of Engineering, Monash University Malaysia \\ Jalan Lagoon Selatan, 47500 Bandar Sunway, Selangor, Malaysia \\ manhvu.tran@monash.edu \\ ${ }^{2}$ The University of Nottingham Malaysia Campus \\ Jalan Broga, 43500 Semenyih, Selangor, Malaysia \\ gianfranco.scribano@nottingham.edu.my
}

\begin{abstract}
Explosion of a fuel/air combustible mixture in a closed system has been recognized as a serious hazard, because it may damage a chamber if there is no proper venting or explosion suppressing device. In the present study, important explosion characteristics of syngas/air flames were investigated in order to evaluate the hazard of the combustion. Results showed the maximum explosion pressure increased from lean (equivalence ratio of 0.8 ) to an equivalence ratio, $\phi$ of 1.2, then decreased significantly with richer mixtures, indicating that maximum explosion pressure occurred at $\phi=1.2$, while explosion time was shortest at $\phi=1.6$. Increasing hydrogen content in the fuel blends significantly raised laminar burning velocity and shortened the explosion time, thereby increasing the maximum rate of pressure rise and deflagration index. Normalized peak pressure, the maximum rate of pressure rise and the deflagration index were sensitive to the initial pressure of the mixture, showing that they increased significantly with increased initial pressure.
\end{abstract}

Keywords: Syngas/air; Maximum peak of pressure; Explosion time; Maximum rate of pressure rise; Deflagration index

\section{Introduction}

Conventional fossil fuels such as oil, natural gas, and coal still supply about $80 \%$ of the world's energy, but due to their limited availability they are expected to be depleted within 40 or 50 years. In the meantime, byproducts from fossil fuel combustion are a major source of environmental problems - the greenhouse effect, ozone depletion and climate change. In this context, syngas has emerged as the likely candidate for greener energy conversion since it possesses numerous advantages in stationary power generation [1,2]. Syngas is formed in a gasification process of coal, biomass, organic wastes and refinery residuals, and it primarily contains hydrogen and carbon monoxide. Depending on the fuel sources and/or processing techniques used to produce it, syngas can also contain nitrogen, carbon dioxide, water, methane and higher-order hydrocarbons $[3,4]$.

In transportation, storage and fuel usage, explosion is a serious hazard that causes injury and damage to its surroundings. When such an explosion takes place in a closed chamber, without proper venting or suppressing devices, it can damage the combustion chamber. To prevent such incidents, it is necessary to understand fuel explosion behavior and its proper use.

Among the combustion characteristics being investigated, maximum rate of pressure rise during explosion in a closed chamber, $(d P / d t)_{\max }$, and the deflagration index, $K_{G}$, are the most important explosion characteristics of premixture combustion. Because $(d P / d t)_{\max }$ depends on both the mixture properties (mixture composition, initial pressure and initial temperature) and the volume of the combustion chamber, $V$, it is normalized with respect to the chamber volume and according to the cubic root law [5] to obtain a deflagration index that is an intrinsic property of the premixture, so that $K_{G}$ is independent of the combustion chamber volume,

$$
K_{G}=(d P / d t)_{\max } \times V^{1 / 3}
$$

The deflagration index is used to evaluate the explosive consequence of a mixture [5]. Other fundamental parameters that characterize the explosion, and will be evaluated here, include maximum explosion pressure, or the highest pressure attained during the explosion, $P_{\max }$, and the explosion time, $t_{c}$, which is defined as the interval of time between ignition and the moment when maximum pressure is reached. 


\section{Experimental setup}

The experimental apparatus consisted of a $6.91 \mathrm{~L}$ (diameter $20 \mathrm{~cm}$, length $22 \mathrm{~cm}$ ) constant volume combustion chamber (CVCC), flow controllers, a pressure transducer, a pressure transmitter and an ignition system, shown schematically in Fig. 1. Component gases of hydrogen, carbon monoxide and air were supplied to the closed chamber at corresponding partial pressures until a desired mixture was obtained. The mixture was left for about 15 minutes to ensure complete blending (by diffusion) and quiescent conditions. The mixture was then ignited at the center of the chamber by the ignition system; from there a flame kernel formed, propagating spherically outward and quenching when it touched the chamber walls [6-8]. Temporal evolution of the pressure during the combustion process was recorded using a piezo-electric pressure transducer (Kistler 6061B), a charge amplifier (Kistler 5011B), a data acquisition device (NI 9215A) and a computer. At least three experiments were performed for each mixture, with a thorough cleaning of the chamber (ventilation of unburned gases, removal of condensed water vapor, cooling to room temperature) carried out between the two consecutive experiments.

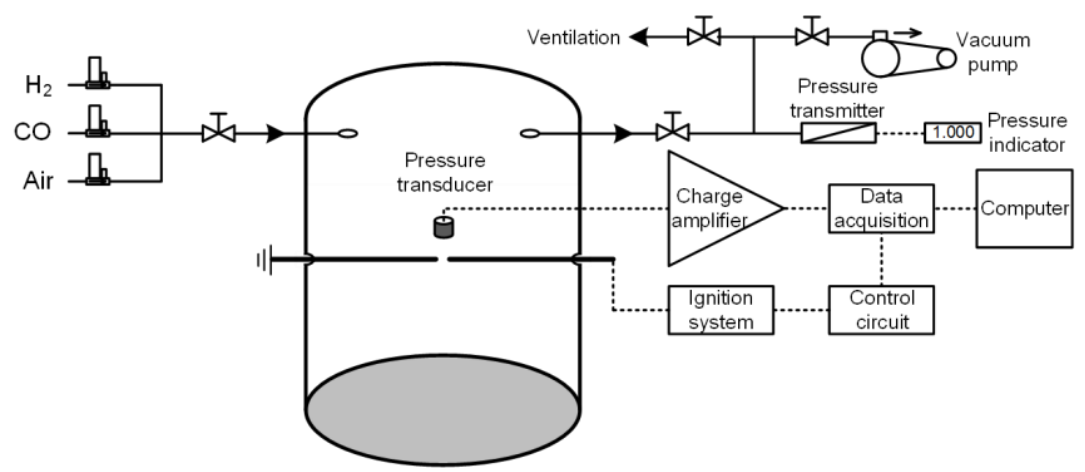

Fig. 1. Schematic of experimental setup.

A typical pressure evolution of an explosion is presented in Fig. 2. The pressure was found to increase gradually during the first couple of milliseconds after ignition. It then increased rapidly within a few subsequent milliseconds. The rate of pressure rise reached its peak value, $(d P / d t)_{\max }$, during this time period; after this peak the pressure was found to further increase, but at a decreasing rate. When $P_{\max }$ was reached, it began to decrease, since most of the component gases were burned out.

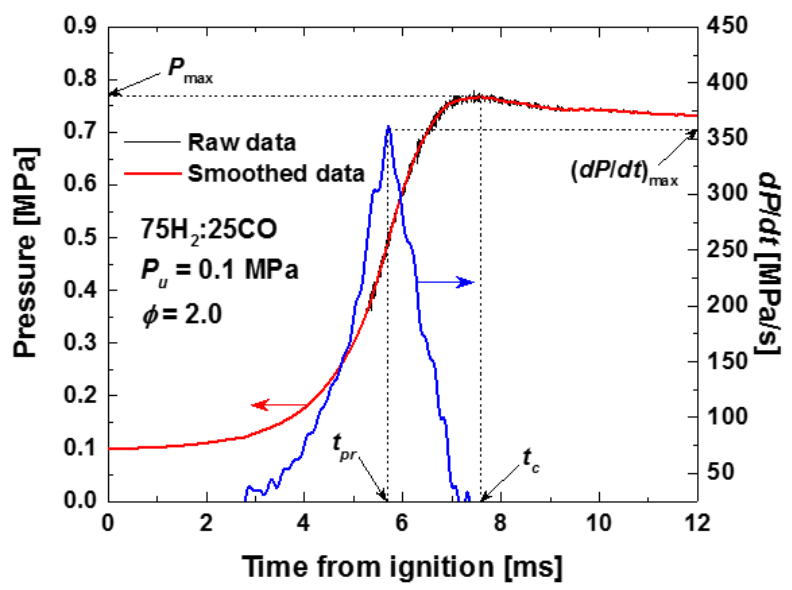

Fig. 2. Pressure (red) and rate of pressure rise (blue) during explosion of $75 \mathrm{H}_{2}: 25 \mathrm{CO} /$ air at $P_{u}=0.1 \mathrm{MPa}$ and $\phi=2.0$. 


\section{Numerical simulation}

The analysis of a gaseous explosion process in a cylindrical vessel was conducted by means of a finite-volume CFD three dimensional model, based on the unsteady Reynolds Averaged Navier Stokes approach. A 3-D cylindrical geometry geometry model, with dimensions identical to the chamber used in the experiment, was generated. The gaseous explosion process followed conservations of mass, momentum, energy and chemical components.

For the turbulence closure model, the realizable $k-\varepsilon$ model, incorporating the solution of transport equations for the turbulent kinetic energy and its dissipation rate, was chosen [9]. The laminar finite-rate model was used to compute chemical source terms. The optimized $\mathrm{H}_{2} / \mathrm{CO}$ mechanism of Davis et al. [10] with 14 species and 30 reactions was used. For density, the ideal gas law was selected. The transport property of pure species was determined based on the molecular kinetic theory; and the specific heat capacity of pure species was evaluated with piecewise temperature polynomials. The momentum term was treated with a second-order upwind scheme, and the pressure and velocity were solved with a SIMPLEC algorithm. All of the governing equations were solved with the unsteady solver available in ANSYS Fluent 16.2.

Figure 3a shows the computational domain with 255,000 total cells and grid spacing of $4 \mathrm{~mm}$. The only boundary condition was the temperature on the wall, which was set to $298 \mathrm{~K}$; during the short sequence of the explosion, the wall temperature could not rise substantially. The wall thickness was set at $0.01 \mathrm{~m}$ and the material used was stainless steel.

The initial conditions were the mass fraction of the mixture, $\mathrm{H}_{2}, \mathrm{CO}, \mathrm{O}_{2}$, and $\mathrm{N}_{2}$, which were changed to correspond with the equivalence ratio. The initial temperature of the mixture was $298 \mathrm{~K}$ and the pressure in the vessel was varied to match the experimental conditions. A sphere patching region of $3 \mathrm{~mm}$ radius, with a temperature of $1500 \mathrm{~K}$, was located at the center of the geometry to ignite the combustible mixture. The pressure value was monitored at a point on the wall (Fig. $3 b)$, to match with the pressure transducer location in the experiment. Data were collected every ten time steps with $2 \mathrm{e}^{-5} \mathrm{~s}$ for each time step. Figure $3 \mathrm{c}$ shows an initial temperature field, which is a small high-temperature-regime, located at the center of the 3D cylindrical geometry.

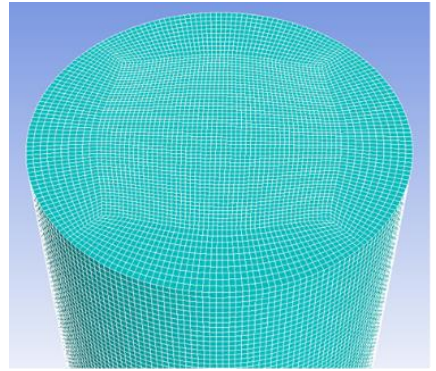

(a)

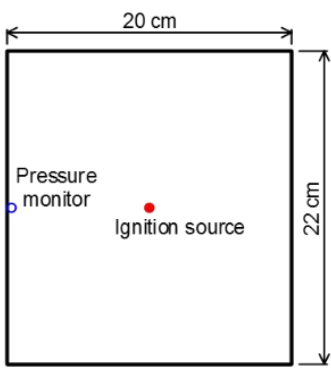

(b)

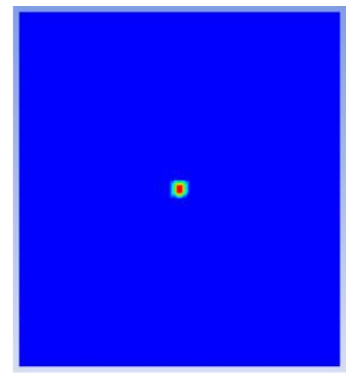

(c)

Fig. 3. CFD model for simulating explosion in the chamber, (a) Mesh of internal fluid, (b) Schematic diagram of chamber, (c) Initial temperature field.

\section{Results and discussion}

\subsection{Temperature and velocity distributions in propagating flame}

Figure 4 shows variations of the simulated temperature and velocity fields of $50 \mathrm{H}_{2}: 50 \mathrm{CO} /$ air propagating flames at initial pressure $\left(P_{u}\right)$ of $0.1 \mathrm{MPa}$ and equivalence ratio $(\phi)$ of 0.8 at different temporal evolutions. In the temperature field shown in Fig. 4a, the red, blue and green (between red and blue) regions represent the burnt, unburned (fresh) gases, and the reacting zone (flame front) respectively. After ignition, the flame front propagated outward spherically until it touched the walls, like the propagating flame observed in the experiment [6-8]. The maximum temperature was around 2200-2500 K. Figure 4b shows that the velocity was greatest at the flame front, where the chemical reaction occurred. It is noteworthy that the fresh gas velocity at the entrance of the preheat zone of the flame front (green color), i.e. the right outward regime of the flame front, was higher than the velocity of the fresh gas far upstream (blue), indicating that the propagating flame pushed away the fresh gas at the preheat zone of the flame front. This phenomenon was reported in a spherically propagating flame [11] and in a propagating edge-flame in a slot burner [12]. 


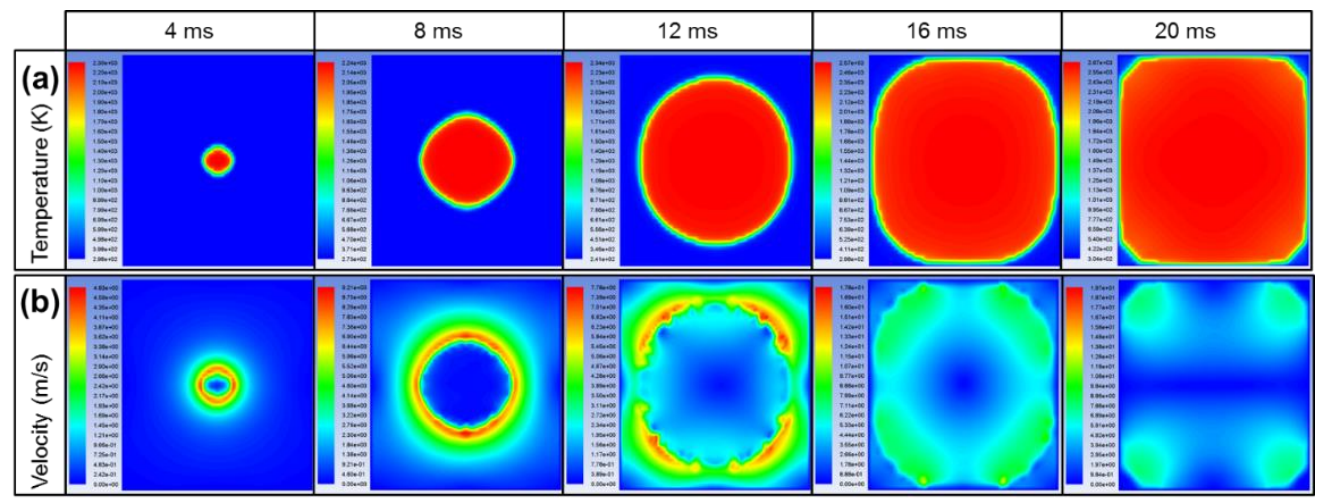

Fig. 4. Simulated (a) temperature and (b) velocity contours of $50 \mathrm{H}_{2}: 50 \mathrm{CO} /$ air propagating flame at $P_{u}=0.1 \mathrm{MPa}$ and $\phi=0.8$.

\subsection{Effect of equivalence ratio}

Figure 5 shows pressure histories during combustion of $50 \mathrm{H}_{2}: 50 \mathrm{CO} /$ air premixed flames for both experimental results (solid curves) and simulated results (dotted curves), at the initial pressure of $0.1 \mathrm{MPa}$ and various equivalence ratios. Both experimental and simulated results showed two notable points: maximum explosion pressure increased from lean $(\phi=0.8)$ to equivalence ratio of 1.2 , then decreased significantly when mixtures were rich, indicating that maximum explosion pressure occurred at $\phi=1.2$. This occurred because the maximum value of the adiabatic flame temperature for $50 \mathrm{H}_{2}: 50 \mathrm{CO} /$ air mixture was at $\phi=1.2$ causing maximum explosion pressure to occur at the same equivalence ratio. Another interesting point is that the shortest explosion time occurred at $\phi=1.6$. The laminar burning velocity of the $50 \mathrm{H}_{2}: 50 \mathrm{CO} /$ air mixtures reached the highest value at $\phi=2.0[13,14]$; therefore, the combination of the two effects - the maximum adiabatic flame temperature at $\phi=1.2$ and the maximum laminar burning velocity at $\phi=2.0$ - caused the explosion time, $t_{c}$, of the $50 \mathrm{H}_{2}: 50 \mathrm{CO} /$ air mixtures to be shortest at $\phi=1.6$.

The pressure history curves of the simulated results show the same trend as the experimental results, which increased rapidly after ignition until reaching maximum explosion pressure after which it decreased. It can be observed that the results of the pressure rise between the experiment and simulation showed good agreement, although the experimental maximum pressure was higher than the simulated results in all cases. Differences in $P_{\max }$ were less pronounced at lean and stoichiometric mixtures which were less than $2 \%$, while for rich mixtures with equivalence ratios up to 2.0 , the differences were around 5-7\%. However, for mixtures with an equivalence ratio of 3.0, the difference was largest with $11 \%$. A discrepancy between experimental and simulated results was also observed for explosion time. The differences for the mixtures at lean to equivalence ratio of 1.6 were around $20 \%$, while there were discrepancies of more than $100 \%$ for very rich mixtures, including $\phi=2.0$ and 3.0. Note that in this study, the same ignition conditions (location, size and temperature) were used to ignite all mixtures, regardless of the fuel/oxidizer ratio. In the future it will be necessary to study the effects of Fluent setting conditions - especially ignition conditions which may affect the propagation of rich flames, which require higher energy to ignite.

\subsection{Effect of $\mathrm{H}_{2}: \mathrm{CO}$ ratio}

Figure 6 shows the pressure history of various syngas/air mixtures at initial pressure of $0.1 \mathrm{MPa}$ and equivalence ratio of 1.0 for both experimental results (solid curves) and simulated results (dotted curves). The simulated results were in good agreement with the experimental results predicting the pressure rise of syngas/air mixtures. It can be observed that the maximum explosion pressure of syngas/air flames slightly increased with increasing $\mathrm{H}_{2}$ content in the $\mathrm{H}_{2} / \mathrm{CO}$ fuel blends. The explosion time was significantly influenced by the $\mathrm{H}_{2} / \mathrm{CO}$ ratio, showing that it was shortened by increasing the $\mathrm{H}_{2}$ content in the fuel blends. The main reason for the shortened ignition time was that the laminar burning velocity of the syngas/air mixture increased quickly with $\mathrm{H}_{2}$ enrichment in the fuel blends, due to the thermal effect of increasing heat release, thereby increasing the adiabatic flame temperature for $\mathrm{H}_{2}$ enrichment $[15,16]$. Although the 
differences in $P_{\max }$ among these mixtures were not significant, it can be expected that the pressure rise rate for mixtures with high $\mathrm{H}_{2}$ content is much greater than with low $\mathrm{H}_{2}$ content mixture, due to faster explosion time.

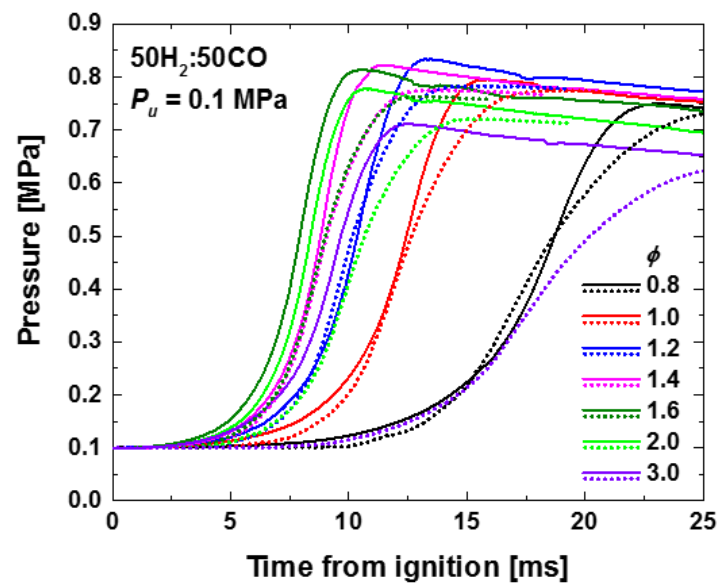

Fig. 5. Explosion pressure of $50 \mathrm{H}_{2}: 50 \mathrm{CO} /$ air mixtures at various equivalence ratios and $P_{u}=$ $0.1 \mathrm{MPa}$. Solid curves represent experimental results, dotted curves are simulated results.

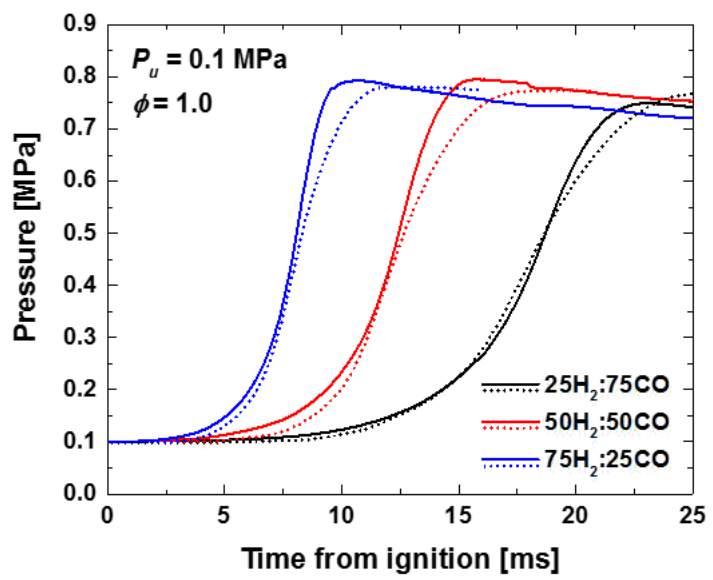

Fig. 6. Explosion pressure of various $\mathrm{H}_{2}$ :CO/air mixtures at $P_{u}=0.1 \mathrm{MPa}$ and $\phi=1.0$. Solid curves represent experimental results, dotted curves are simulated results.

The experimental maximum explosion pressure and the explosion time of various syngas/air flames at $0.1 \mathrm{MPa}$ vs. equivalence ratio are plotted in Fig. 7, which shows that $P_{\max }$ is greatest at $\phi=1.2$ while $t_{c}$ is shortest at $\phi=1.6$ for all syngas/air flames. For a given equivalence ratio, $P_{\max }$ and $t_{c}$ respectively increased and decreased with increased $\mathrm{H}_{2}$ in the syngas mixtures. Figure 8 shows the experimental maximum rate of pressure rise, $(d P / d t)_{\max }$, and the deflagration index, $K_{G}$, of various syngas/air premixed flames vs. equivalence ratio at $P_{u}=0.1 \mathrm{MPa}$. It can be observed that $(d P / d t)_{\max }$ and $K_{G}$ significantly increased with $\mathrm{H}_{2}$ enrichment in the syngas mixtures because of the reduced explosion time, as displayed in Figs. 6 and 7. This indicates that syngas/air premixed flame with higher $\mathrm{H}_{2}$ content has more explosion hazard potential than a syngas mixture with low $\mathrm{H}_{2}$ concentrations. In addition, the maximum of $(d P / d t)_{\max }$ and $K_{G}$ of all syngas/air flames occurred at equivalence ratio of 1.6, corresponding to the shortest $t_{c}$ occurring at the same equivalence ratio.

\subsection{Effect of initial pressure}

Figure 9 shows normalized pressures $\left(P / P_{u}\right)$ of the $50 \mathrm{H}_{2}: 50 \mathrm{CO} /$ air premixed flames at equivalence ratio of 0.8 , with various initial pressures. It can be observed that pressure rise during combustion is very sensitive to the initial pressure change of the mixture. With the initial pressure elevation, the maximum explosion pressure increased and the explosion time became shorter, significantly increasing the pressure rise rate. Compared to experimental results, the simulated pressure rise showed good agreement for the flame at $P_{u}=0.1 \mathrm{MPa}$, and acceptable agreement at $P_{u}=0.15 \mathrm{MPa}$; however, the discrepancies became greater when the initial pressure was increased. In the range of the initial pressure tests, the greatest difference between experimental and simulated results in $P_{\max }$ was $12.3 \%$, and in $t_{c}$ was $68 \%$ at initial pressure of $0.4 \mathrm{MPa}$, noting that explosion time discrepancy began at the early simulation stage, when the flame took nearly $12 \mathrm{~ms}$ to begin propagating, although the pressure rise rates were almost the same. In similar, very rich flames $(\phi>2.0)$ shown in Fig. 5, the ignition conditions could have a strong influence in the early propagating flame, affecting the explosion time of the flame at elevated initial pressures. 


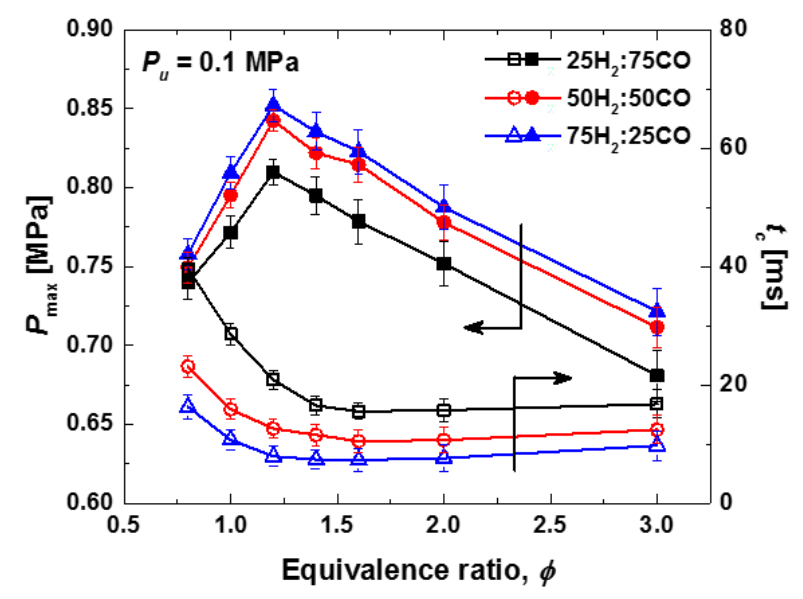

Fig. 7. Experimental maximum explosion pressure and explosion time of various $\mathrm{H}_{2}$ :CO/air mixtures vs. equivalence ratio at $P_{u}=0.1 \mathrm{MPa}$.

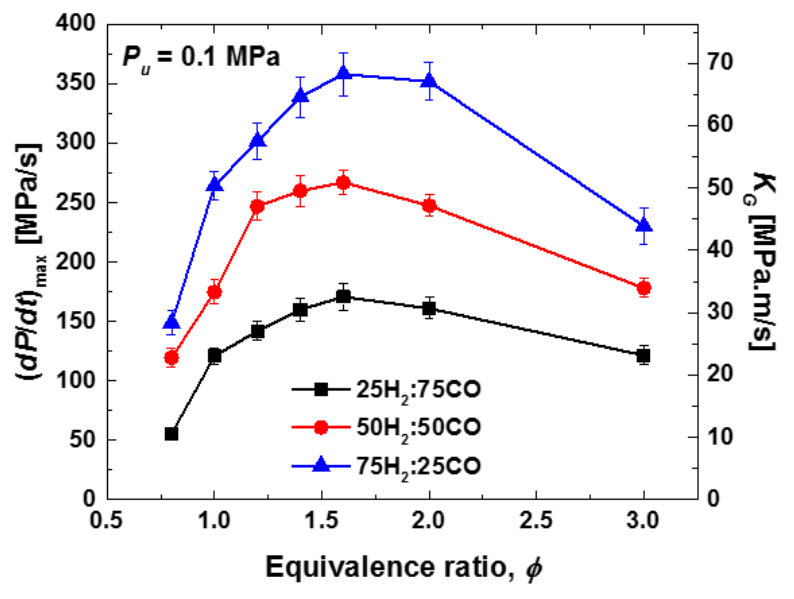

Fig. 8. Experimental maximum rate of pressure rise and deflagration index of various $\mathrm{H}_{2}$ :CO/air mixtures vs. equivalence ratio at $P_{u}=0.1 \mathrm{MPa}$.

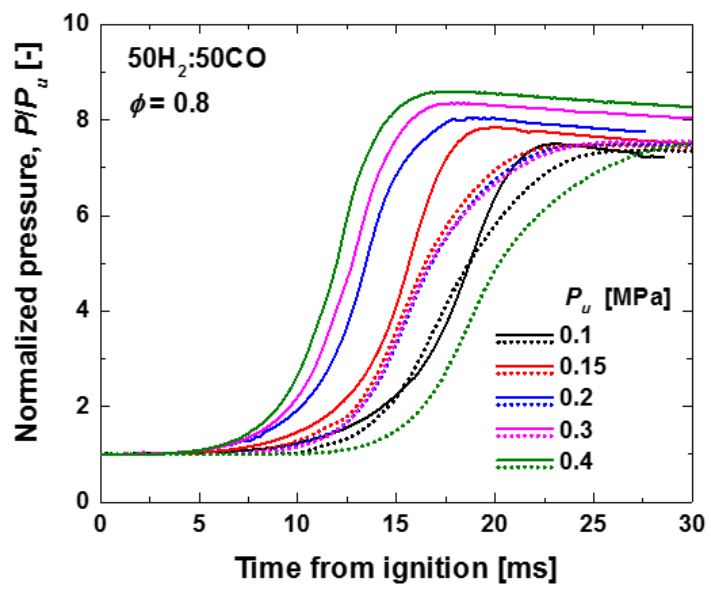

Fig. 9. Explosion pressure of $50 \mathrm{H}_{2}: 50 \mathrm{CO} /$ air mixtures at various initial pressures and $\phi=0.8$. Solid curves represent experimental results, dotted curves are simulated results.

Figure 10 shows experimental normalized peak pressures $\left(P_{\max } / P_{u}\right)$ and explosion time of $25 \mathrm{H}_{2}: 75 \mathrm{CO} /$ air premixed flames at various initial pressures. Normalized peak pressures were plotted to easily compare the maximum explosion pressure of mixtures at different initial pressures. It is clearly demonstrated that $P_{\max } / P_{u}$ increased as initial pressure grew, due to the rise of adiabatic flame temperature with the initial pressure elevation. Higher initial pressure caused a more serious explosion hazard, which agrees with results obtained in the past [17].

However, results observed in this study, indicating that shorter explosion time occurs with initial elevated pressure, are contradictory to the results in Ref. [17]. It is noted that unstretched laminar burning velocity, $S_{L}^{0}$, was reduced with the increase of initial pressure. Therefore, the explanation for the influence of $S_{L}^{0}$ on explosion time is not applicable to propagating flame at elevated initial pressures conditions. It was reported that in order to avoid the influence of spark ignition and wall interference, and to limit the pressure increase to within $1 \%$ of the initial pressure, only small propagating flames (radius larger than $6 \mathrm{~mm}$ and smaller than $30 \mathrm{~mm}$ in Refs. [6-8]) were considered in measuring the $S_{L}{ }^{0}$ with the outwardly propagating spherical flame method. As shown in Fig. 9, flame with radii in the range considered to calculate $S_{L}{ }^{0}$ corresponded to the first couple of milliseconds after the ignition. It can be seen that the small propagating 
flames did not influence the explosion time. Instead, self-acceleration of the flame, caused by flame front instabilities at elevated pressures conditions, was the dominant factor in turbulent propagating flames [18]. With the increased initial pressure, caused by the promotion of hydrodynamic instability from the substantial decrease of the flame thickness, the formation of cellular instabilities produced a corrugated flame front that could induce the turbulence of unburned mixture, mixture, and subsequently, the rapid increase of flame propagation velocity [6-8], the main reason for the shortened explosion time. The experimental maximum rate of pressure rise, $(d P / d t)_{\max }$, and the deflagration index, $K_{G}$, of the $25 \mathrm{H}_{2}: 75 \mathrm{CO} / \mathrm{air}$ premixed flames vs. the equivalence ratio at different initial pressures are plotted in Fig. 11 . The results clearly indicated that $(d P / d t)_{\max }$ and $K_{G}$ were significantly increased with the increase of the initial pressure of mixtures. With the increased initial pressure, a faster turbulent flame propagation caused a shorter explosion time and hence a higher rate of pressure rise, corresponding to a higher deflagration index.

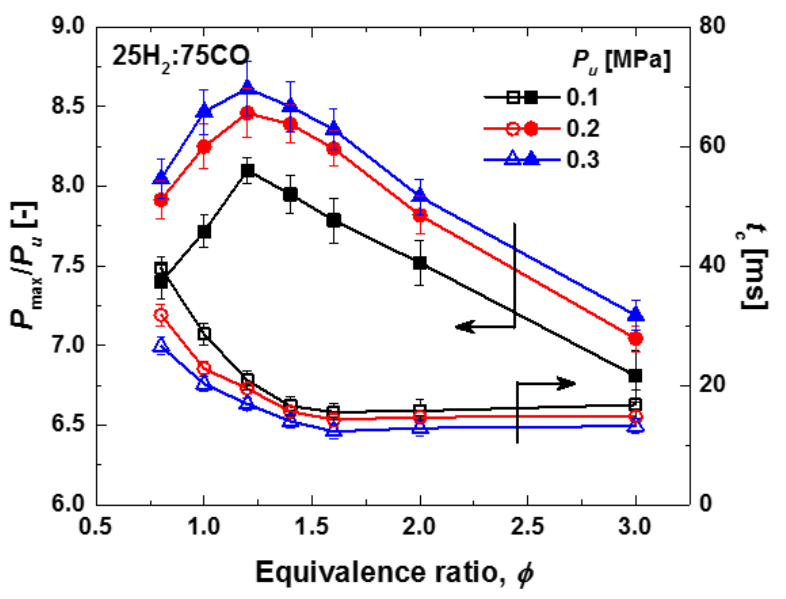

Fig. 10. Experimental normalized peak pressure and explosion time of $25 \mathrm{H}_{2}: 75 \mathrm{CO} /$ air mixtures vs. equivalence ratio at various initial pressures.

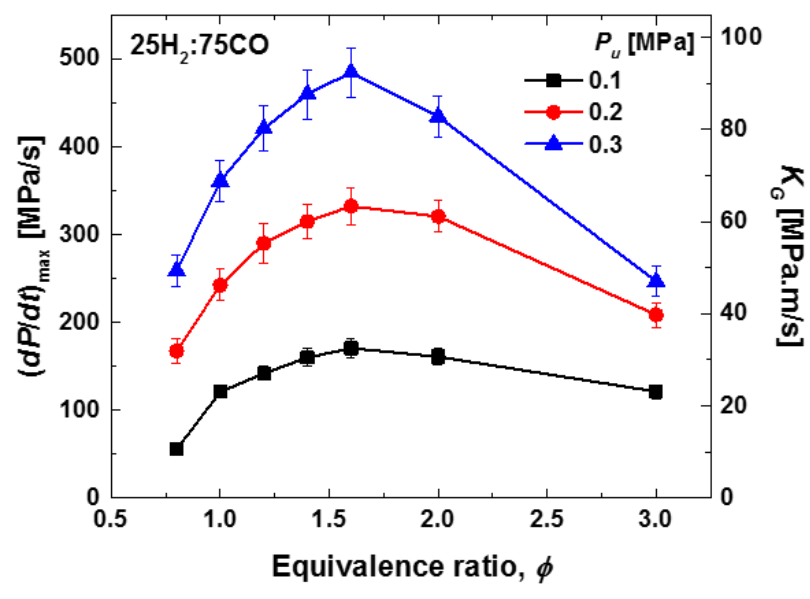

Fig. 11. Experimental maximum rate of pressure rise and deflagration index of $25 \mathrm{H}_{2}: 75 \mathrm{CO} /$ air mixtures vs. equivalence ratio at various $P_{u}$.

\section{Conclusion}

In this study, explosion characteristics such as maximum explosion pressure, $P_{\max }$, explosion time, $t_{c}$, maximum rate of pressure rise, $(d P / d t)_{\max }$, and deflagration index, $K_{G}$, of various syngas/air flames at different equivalence ratios and initial pressures, were investigated experimentally and numerically to evaluate the hazard of the explosion of syngas/air mixtures. The major conclusions of the study are as follows:

1. Because adiabatic flame temperature is maximized at $\phi=1.2$, it causes the maximum explosion pressure to occur at the same equivalence ratio; while the combination of maximum adiabatic flame temperature at $\phi=1.2$ and maximum laminar burning velocity at $\phi=2.0$ results in the shortest explosion time at $\phi=1.6$.

2. The maximum rate of pressure rise and the deflagration index of syngas/air premixed flames significantly increase with the enhancement of $\mathrm{H}_{2}$ concentration in the fuel blend, which shortens the explosion time. Therefore, the syngas mixture with high $\mathrm{H}_{2}$ content has a higher potential explosion hazard than a syngas mixture with low $\mathrm{H}_{2}$ concentration.

3. Normalized peak pressure increases with increased initial pressure. The maximum rate of pressure rise and the deflagration index also increase with the initial pressure because of enhanced adiabatic flame temperature and hydrodynamic instability, causing an increase in turbulent propagating flame velocity at elevated pressures.

4. Simulated results using ANSYS Fluent showed good agreement with experimental results for most equivalence ratios at atmospheric pressure. However, large discrepancies were observed for very rich mixtures $(\phi>2.0)$ and at elevated pressure conditions. The effects of Fluent setting conditions must be studied further. 


\section{References}

[1] K. H. Casleton, R. W. Breault, G. A. Richards, "System issues and tradeoffs associated with syngas production and combustion," Combust. Sci. Technol., vol. 180, pp. 1013-1052, 2008.

[2] Y. Richardson, J. Blin, A. Julbe, "A short overview on purification and conditioning of syngas produced by biomass gasification: Catalytic strategies, process intensification and new concepts," Prog. Energy. Combust. Sci., vol. 38, pp. 765-781, 2012.

[3] J. Natarajan, T. Lieuwen, J. Seitzman, "Laminar flame speeds of $\mathrm{H}_{2} / \mathrm{CO}$ mixtures: Effect of $\mathrm{CO}_{2}$ dilution, preheat temperature, and pressure," Combust. Flame, vol. 151, pp. 104-119, 2007.

[4] J. S. Ha, J. Park, T. M. Vu, O. B. Kwon, J. H. Yun, S. I. Keel, "Effect of flame stretch in downstream interaction between premixed syngas-air flames," Int. J. Hydrogen Energy, vol. 36, pp. 13181-13193, 2011.

[5] NFPA 68, Guide for Venting Deflagrations, 2013 ed., National Fire Protection Association, 2013.

[6] T. M. Vu, J. Park, O. B. Kwon, J. S. Kim, "Effects of hydrocarbon addition on cellular instabilities in expanding syngasair spherical premixed flames," Int. J. Hydrogen Energy, vol. 34, pp. 6961-6969, 2009.

[7] T. M. Vu, J. Park, O. B. Kwon, D. S. Bae, J. H. Yun, S. I. Keel, "Effects of diluents on cellular instabilities in outwardly propagating spherical syngas-air premixed flames," Int. J. Hydrogen Energy, vol. 35, pp. 3868-3880, 2010.

[8] T. M. Vu, J. Park, J. S. Kim, O. B. Kwon, J. H. Yun, S. I. Keel, "Experimental study on cellular instabilities in hydrocarbon/hydrogen/carbon monoxide-air premixed flames," Int. J. Hydrogen Energy, vol. 36, pp. 6914-6924, 2011.

[9] B. E. Launder, D. B. Spalding, "Mathematical Models of Turbulence," New York: Academic Press; 1972.

[10] S. G. Davis, A. V. Joshi, H. Wang, F. Egolfopoulos, "An optimized kinetic model of $\mathrm{H}_{2} / \mathrm{CO}$ combustion," Proc. Combust. Inst., vol. 30, pp. 1283-1292, 2005.

[11] E. Varea, V. Modica, A. Vandel, B. Renou, "Measurement of laminar burning velocity and Markstein length relative to fresh gases using a new postprocessing procedure: Application to laminar spherical flames for methane, ethanol and isooctane/air mixtures," Combust. Flame, vol. 159, pp. 577-590, 2012.

[12] M. -V. Tran, M. S. Cha, "Correction of edge-flame propagation speed in a counterflow, annular slot burner," Combust. Flame, vol. 162, pp. 4671-4672, 2015.

[13] I. C. McLean, D. B. Smith, S. C. Taylor, "The use of carbon monoxide/hydrogen burning velocities to examine the rate of the CO + OH reaction," Proc. Combust. Inst., vol. 25, pp. 749-757, 1994.

[14] H. Sun, S. I. Yang, G. Jomaas, C. K. Law, "High-pressure laminar flame speeds and kinetic modeling of carbon monoxide/hydrogen combustion," Proc. Combust. Inst., vol. 31, pp. 439-446, 2007.

[15] O. Park, P. S. Veloo, H. Burbano, F. N. Egolfopoulos, "Studies of premixed and non-premixed hydrogen flames," Combust. Flame, vol. 162, pp. 1078-1094, 2015.

[16] V. Amin, G. Katzlinger, P. Saxena, E. Pucher, K. Seshadri, "The influence of carbon monoxide and hydrogen on the structure and extinction of nonpremixed and premixed methane flames," Proc. Combust. Inst., vol. 35, pp. 955-963, 2015.

[17] Q. Li, Y. Cheng, Z. Huang, "Comparative assessment of the explosion characteristics of alcohol-air mixtures," J. Loss Prev. Process Ind., vol. 37, pp. 91-100, 2015.

[18] O. Mannaa, M. S. Mansour, W. L. Roberts, S. H. Chung, "Laminar burning velocities at elevated pressures for gasoline and gasoline surrogates associated with RON," Combust. Flame, vol. 162, pp. 2311-2321, 2015. 